\title{
Decision-Oriented Multi-Outcome Modeling for Anesthesia Patients
}

\author{
Zhibin Tan ${ }^{1}$, Romeo Kaddoum ${ }^{2}$, Le Yi Wang*, ${ }^{1}$ and Hong Wang ${ }^{2}$ \\ ${ }^{I}$ Dept. of Electrical and Computer Engineering, Wayne State University, Detroit, Michigan 48202, USA \\ ${ }^{2}$ Dept. of Anesthesiology, Wayne State University, Detroit, Michigan 48201, USA
}

\begin{abstract}
Anesthesia drugs have impact on multiple outcomes of an anesthesia patient. Most typical outcomes include anesthesia depth, blood pressures, heart rates, etc. Traditional diagnosis and control in anesthesia focus on a one-drugone-outcome scenario. This paper studies the problem of real-time modeling for monitoring, diagnosing, and predicting multiple outcomes of anesthesia patients. It is shown that consideration of multiple outcomes is necessary and beneficial for anesthesia managements. Due to limited real-time data, real-time modeling in multi-outcome modeling requires lowcomplexity model strucrtures. This paper introduces a method of decision-oriented modeling that significantly reduces the complexity of the problem. The method employs simplified and combined model functions in a Wiener structure to contain model complexity. The ideas of drug impact prediction and reachable sets are introduced for utility of the models in diagnosis, outcome prediction, and decision assistance. Clinical data are used to evaluate the effectiveness of the method.
\end{abstract}

Keywords: Modeling, anesthesia, multi-outcome, diagnosis, prediction, control.

\section{INTRODUCTION}

Real-time anesthesia decisions are exemplified by general anesthesia for attaining an adequate anesthetic depth (consciousness level of a patient), ventilation control, etc. One of the most critical requirements in this decision process is to predict the impact of the inputs (drug infusion rates, fluid flow rates, etc.) on the outcomes (consciousness levels, blood pressures, heart rates, etc.). This prediction capability can be used for control, display, warning, predictive diagnosis, decision analysis, outcome comparison, etc. The core function of this prediction capability is embedded in establishing a reliable model that relates the drug or procedure inputs to the outcomes. Traditional modeling, diagnosis, and control in anesthesia focus on a one-drug-oneoutput scenario [1-7]. Typically, an anesthesia drug influences more than one patient outcomes. For monitoring, diagnosis, and control, it becomes essential that the impact of anesthesia drugs on multiple outcomes be taken into consideration. It has also been observed that each patient responses to drug inputs with very different dynamics. Even for the same patient, responses to the same drugs change with time, surgical stages, and patient conditions. As a result, it is necessary to establish multi-input-multi-output (MIMO) models in real-time and for individual patients.

Due to limited real-time data, individualized real-time patient modeling must have low model complexity. As a result, the task of real-time modeling in multi-drug-multioutcome modeling is of substantial challenge in complexity reduction. A basic information-oriented model structure (a special case of Wiener models) was introduced in [8-11], for patient anesthesia depth responses to propofol infusion as a

*Address correspondence to this author at the Dept. of Electrical and Computer Engineering, Wayne State University, Detroit, Michigan 48202, USA; Tel: 313-577-4715; Fax: 313-578-5834; E-mail: lywang@wayne.edu single-input-single-output (SISO) system. This simple model structure contained only a few parameters and could be easily identified in real-time. This paper introduced a method of decision-oriented MIMO modeling that significantly reduced the complexity of the problem. The method employed simplified and combined model functions in a Wiener structure to contain model complexity. The ideas of drug impact prediction and reachable sets were introduced for utility of the models in diagnosis, outcome prediction, and decision assistance. Clinical data were used to evaluate the effectiveness of the method.

\section{DATA ACQUISITION}

The patient population age group was between 20 and 70 years old. These patients were undergoing upper extremity arteria-venous fistula placement or thrombectomy, under intravenous unconscious sedation. Anesthesia was administered by an experienced anesthesiologist or registered nurse anesthetist. The patient was seen, examined and evaluated in the pre-operative holding area by an anesthesiologist. The anesthesiologist made sure that the patient was ready for the surgery. Labs were checked in the pre-operative holding area and $1 \mathrm{mg}$ of Midazolam IV was administered to the patient, after receiving full consent for the surgery and the participation in this study. All risks and benefits were thoroughly explained to the patient while obtaining consent.

The patient was, then, taken to the operating room, placed on the OR table, started on face mask oxygen at a rate of $8 \mathrm{~L} / \mathrm{min}$, hooked to the electrocardiogram monitor, noninvasive blood pressure cuff was placed on the contralateral arm, and the cuff cycle was set to measure blood pressure every three minutes. A pulse oximeter was hooked on the patient's contralateral index.

The patient consciousness levels during anesthesia were measured by a BIS (bi-spectrum) monitor (Aspect Medical 
Devices, Inc.). It was one of the anesthesia monitors commercially available and widely used in operation rooms $[12,13]$. The monitor provided continuously an index in the range of $[0,100]$ such that the lower the index value, the deeper the anesthesia state. Hence, an index value 0 will indicate "brain dead" and 100 will be "awake." A bispectral (BIS) electrode was placed on the patient's forehead before administering anesthesia to the patient. The electrode was connected to the BIS monitor, which in turn was connected to a special computer system to allow continuous recording and saving of the BIS values. The computer's software was a monitoring system designed by the Department of Electrical and Computer Engineering at Wayne State University. The system performed prediction of BIS values for the specific patient by generating patient models in real time using response data from the patient under anesthesia, see Figs. (1 and 2).

A baseline BIS value of at least 90 was recorded before the administration of anesthesia. The patient was given 1-2 $\mathrm{mcg} / \mathrm{kg}$ of bolus IV Fentanyl at the beginning of the surgery and $1 \mathrm{mcg} / \mathrm{kg}$ bolus during the surgery as needed. The patient was started on intravenous propofol pump at a rate of $50 \mathrm{mcg} / \mathrm{kg} / \mathrm{min}$ and titrated as needed during the surgery. All measured heart rates, blood pressures and pulse oximetry values were entered and saved manually into the computer every three minutes and following any bolus administrations. The propofol rate, any changes made to the propofol rate, and any propofol or Fentanyl bolus given were transmitted to the computer monitoring system automatically and continuously at the sampling rate of $1 \mathrm{~Hz}$ (one sample per second). Towards the end of the procedure, and after making sure no more surgical stimuli were applied to the patient, all anesthetics were turned off and the patient was awakened with the BIS value of more than 75 . The patient was then taken to the recovery room on oxygen tank for a period of two hours of observation.

As a pilot study for methodology development, anesthesia procedure data from 5 patients were collected. The data set was not large enough for reliable statistical analysis, but provided sufficient data for developing our model structures and algorithms and to evelaute their potential advantages.

\section{RATIONALE FOR MIMO, REAL-TIME, AND INDIVIDUALIZED MODELING OF PATIENT DYNAMICS}

In this section, we provide several reasons why it is important to consider multiple outcomes simultaneously,

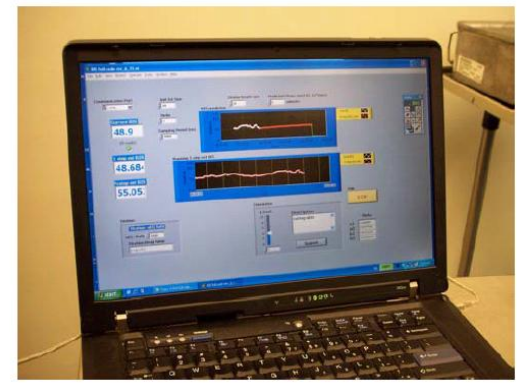

Fig. (1). Computer data acquisition system.

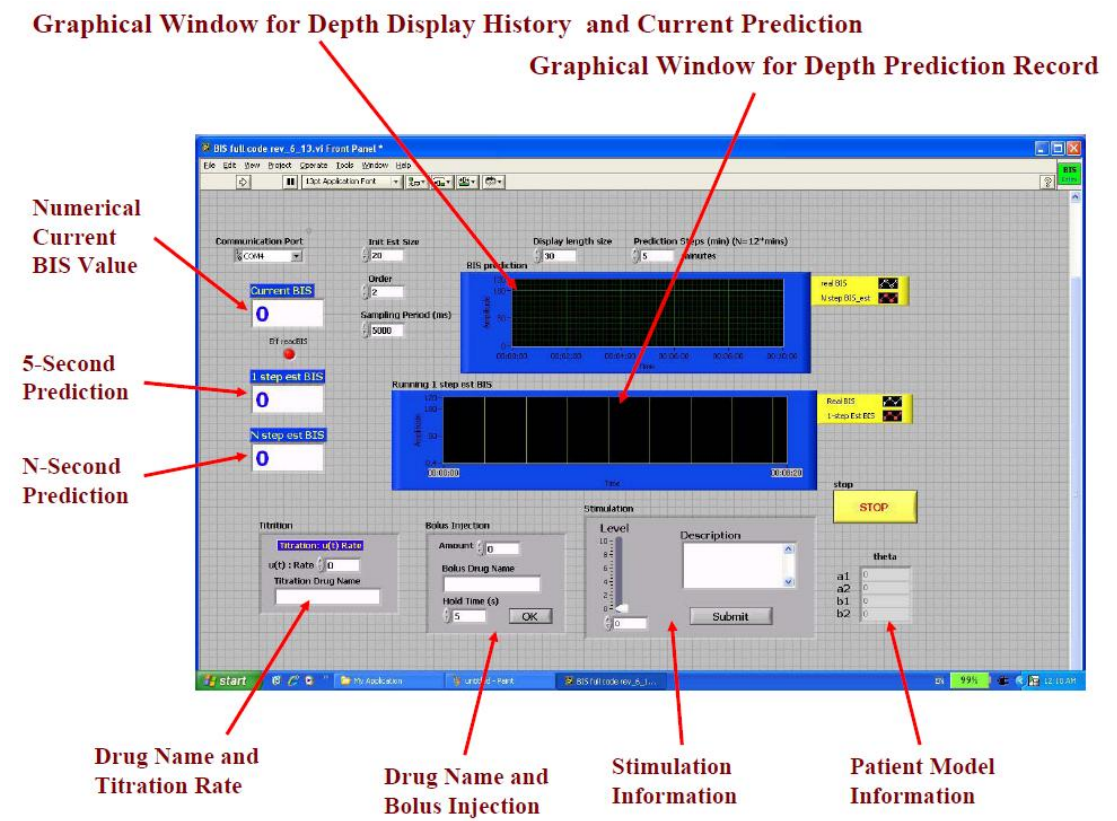

Fig. (2). Front panel of the system. 
establish individualized models, and use real-time data to capture patient responses.

Typically, an anesthesia drug influences more than one patient outcomes. Fig. (3) shows a typical recording of a patient's response to propofol and fentanyl titration and bolus injections. For this patient, the anesthesia drugs not only reduced the patient BIS values to a lower level, but also depressed the blood pressure and made the heart rate fluctuate. For monitoring, diagnosis, and control, it becomes essential that the impact of anesthesia drugs on both anesthesia depth and blood pressures be taken into consideration.

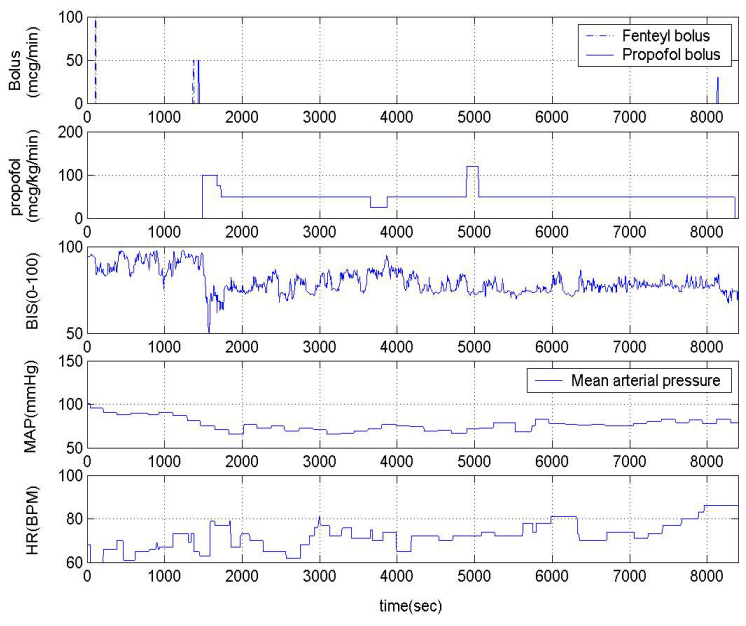

Fig. (3). Drug inputs influence many patient outcomes.

\subsection{Individualized and Time Varying Patient Dynamics}

Each patient responses to drug inputs with very different dynamics. Fig. (4) is another patient's response to the same types of anesthesia drugs as in Fig. (3). In comparison to Fig. (3), this patient demonstrated slower response after drug changes or bolus injections, lower sensitivity near steady state, and more heart rate variations during the process. For example, this patient's BIS index reached the steady state value of 75 after 750 seconds, in comparison to the steady state BIS value of 60 after around 1600 seconds in the patient data in Fig. (3). Similar disparity was also shown in blood pressures. In Fig. (4), the blood pressure dropped to the value around $90 \mathrm{mmHg}$, in comparison to the value of 65 $\mathrm{mmHg}$ in Fig. (3). Consequently, to improve accuracy in anesthesia management, it is necessary to obtain individualized patient models.

Even for the same patient, responses to the same drugs change with time and surgical stages, and patient conditions. The patient in Fig. (5) initially had a more sensitive response in BIS values to propofol infusion, see the BIS trajectory in the first 100 seconds in which the BIS value dropped from 100 to 65 after the propofol rate was increased to $75 \mathrm{mcg} / \mathrm{kg} / \mathrm{min}$. However, late in the time interval of 240-310 seconds, the BIS values became higher, around 75 , even though the same rate of propofol was administered.

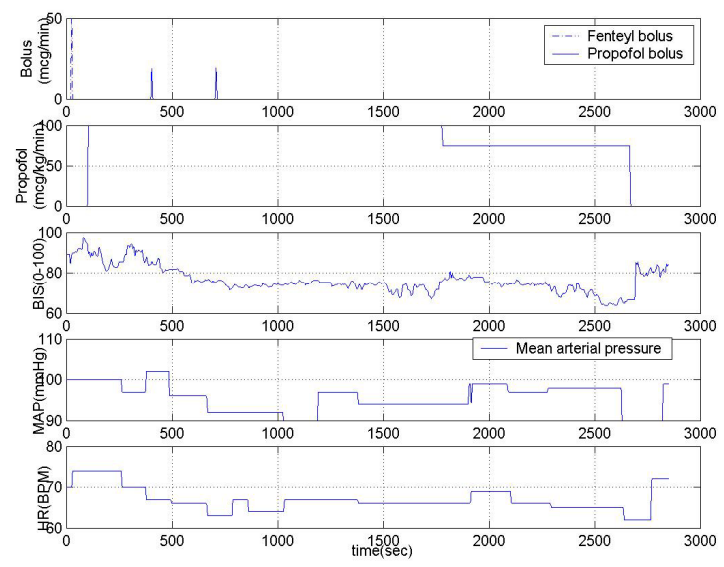

Fig. (4). Different patients demonstrate different dynamics.

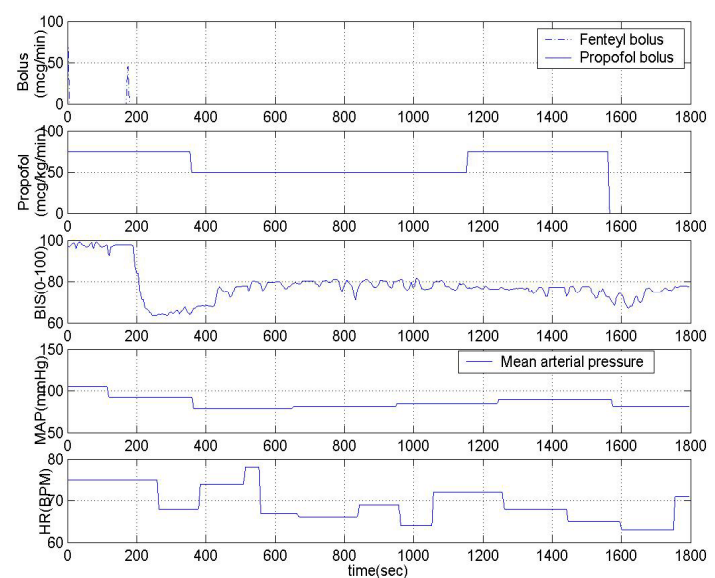

Fig. (5). Patient dynamics change with time.

\subsection{Multiple Drugs and Multi-Objective Anesthesia Administration}

Our case studies involved both propofol and fentanyl. In Fig. (5), both drugs impacted multiple outcomes, although to a different degree. For instance, fentanyl had direct influence on blood pressure while it had no obvious influence on BIS values. In the time interval of 0-200 seconds, the initial injection of fentanyl bolus depressed the blood pressure from 110 to 90 , while there were no obvious changes on BIS values. The propofol input controlled both anesthesia depth and blood pressure significantly. During the time interval of 350-1000 seconds, both of BIS value and blood pressure climbed up as the propofol titration rate was decreased.

Anesthesia management must consider all essential patient outcomes. For instance, if one focuses only on the anesthesia depth, propofol will be increased when the BIS is too high. However, if this occurs when a patient's blood pressures are low and if the patient's blood pressures respond to propofol sensitively, a much more cautious control action will be preferred since aggressive propofol increase may drive blood pressures to an alarming level. Consequently, a 
multi-objective control strategy can potentially deliver a better anesthesia management. Some researchers investigated the problems of multi-variable feedback control with applications in anesthesia [14-17]. As a promising control strategy for regulation of anesthesia patient outcomes, model predictive control was applied to regulate two patient outcomes simultaneously [17]. Most of the previous work concentrated on population based models.

To understand the importance of the multi-objective anesthesia modeling and control, we made a comparison of control actions between the regulation of two patient outcomes (BIS and blood pressure) and the regulation of one patient outcome only. Fig. (6) illustrates the simulation results which were produced by the MATLAB function "scmpc" in the model predictive control toolbox. The data used for simulation were collected real-time patient data in operating rooms. The control actions (the propofol titration rate) were very different in the two cases.
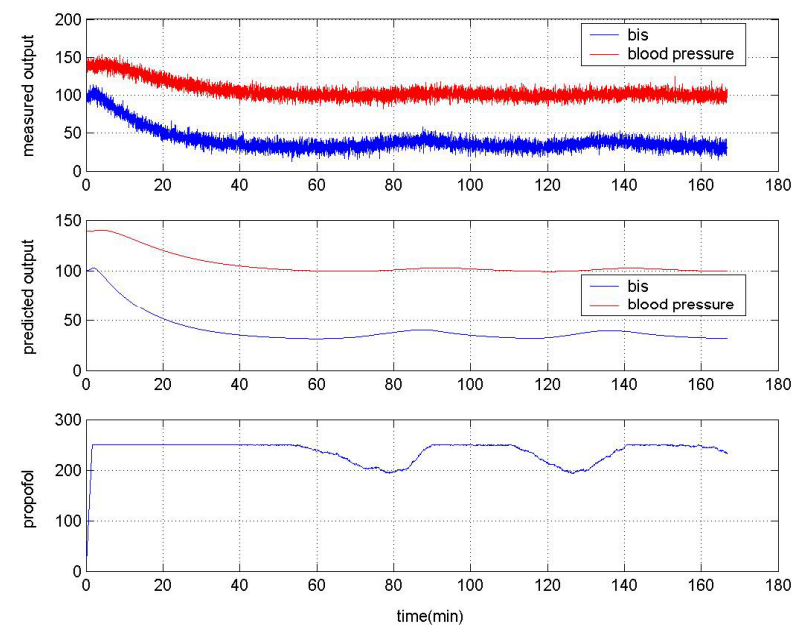

(a)
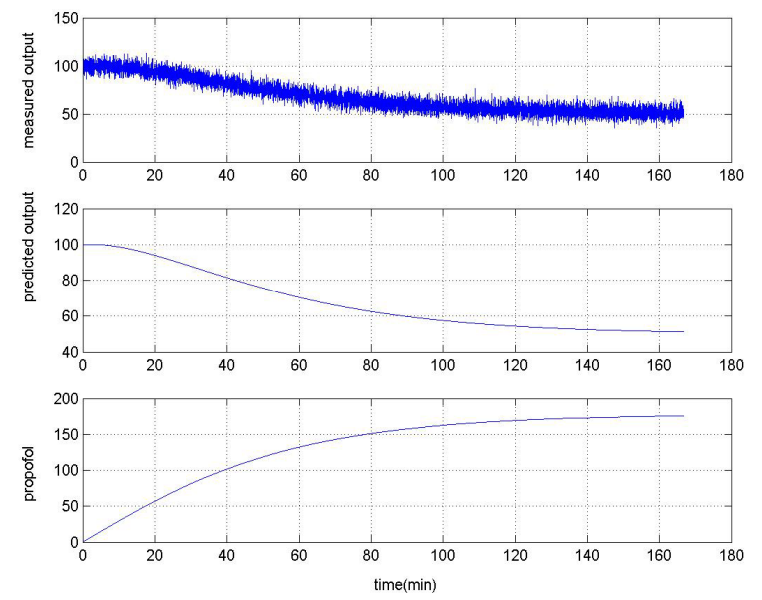

(b)

Fig. (6). Anesthesia managements differ when multiple outcomes were considered. (a) Propofol control based on a combined performance criterion on BIS and blood pressures. (b) Propofol control based on BIS only.
The above discussions indicated that for enhanced anesthesia monitoring and control, it was necessary and beneficial to consider a patient as a multi-input-multi-output dynamic system whose characteristics changed substantially among different patients and over different time intervals.

However, MIMO systems contain far more parameters than single-input-single-output systems. In turn they require more input excitations to ensure model identifiability. A model is identifiable from a given set of data if the data can generate a unique estimate of the system. When data are not sufficiently rich in their information contents, they are not sufficient to generate a unique model. Consequently, parameters cannot be decided. It is well known [18] that the more the parameters the more rich information the data must contain. Input information richness can be enhanced by changing input values frequently, which are commonly characterized as "persistent excitation" conditions. Unfortunately, in anesthesia applications, the input is propofol titration which cannot be arbitrarily modified for modeling purposes. This implies that it is not only highly desirable but in fact necessary to reduce model complexity as much as possible.

\section{MIMO PATIENT MODELING FOR ANESTHESIA MONITORING AND CONTROL}

A basic information-oriented model structure (a special case of Wiener models), for patient anesthesia depth responses to propofol infusion as an SISO system was introduced in [8-11]. This model can also be applied to relate other patient outcomes, such as blood pressure and heart rate, to input drugs. The basic idea from [8-11] is summarized below.

\subsection{Wiener Model Structure}

Propofol titration was administered by an infusion pump. The dynamics of a patient's BIS response to a drug infusion could be divided into several blocks. The response from the titration command to the drug infusion at the needle point was the infusion pump dynamics and could be represented by a transfer function $G_{i}(s)$. Similarly, the BIS monitor dynamics could be represented by a transfer function $G_{m}(s)$.

The patient dynamics was a nonlinear system. Although the actual physiological and pathological features of the patient required models of high complexity, for prediction or control purposes it was not only convenient but essential to use simple models as long as they were sufficiently rich to represent the most important properties of the patient response. Understanding the information used by anesthesiologists in infusion control, we characterized the patient response to propofol titration with three basic components: (1) Initial time delay $\tau_{p}$ after drug infusion: During this time interval after a change of the infusion rate, the BIS value did not change due to the time required for drugs to reach the target tissues and to complete volume distribution. (2) Dynamic reaction: This reflected how fast the BIS value would change once it started to respond, and was modeled by a transfer function $G_{p}(s)$. (3) A nonlinear 
static function for sensitivity of the patient to a drug dosage at steady state: This was represented by a function or a lookup table $f$. The meaning of these system blocks was illustrated in Fig. (7). Combined with infusion pump and monitor models, this model structure for titration response was a special case of the Wiener models shown in Fig. (8).

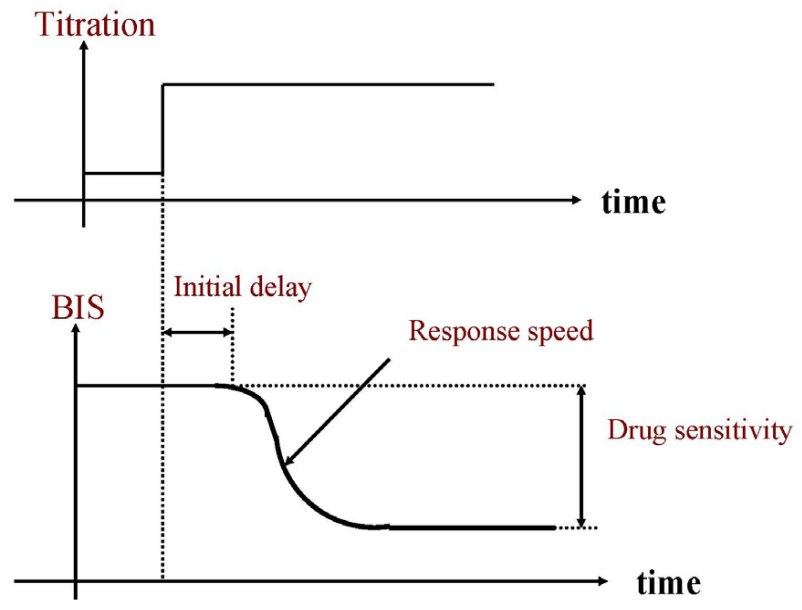

Fig. (7). Simplified patient model structure.

Wiener Model Structure

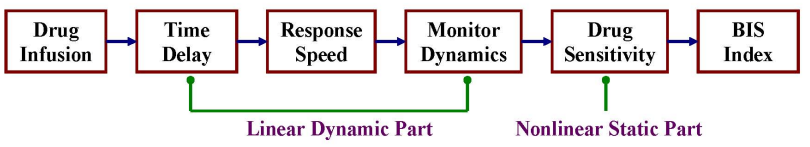

Fig. (8). Wiener model structure.

To establish patient models for monitoring and control, clinical data were collected. One of these data sets was used in this paper. The anesthesia process lasted about 76 minutes, starting from the initial drug administration and continuing until last dose of administration. Propofol was used in both titration and bolus. Fentanyl was injected in small bolus amount three times, two at the initial surgical preparation and one near incision. Analysis showed that the impact of Fentanyl on the BIS values was minimal. As a result, it was treated as a disturbance and not explicitly modeled in this example. The drug infusion was controlled manually by an experienced anesthesiologist. The trajectories of titration (in $\mathrm{mcg} / \mathrm{sec}$ ) and bolus injection (converted to $\mathrm{mcg} / \mathrm{sec}$ ) during the entire surgical procedure were recorded, which are shown together with the corresponding BIS values in Fig. (10). The patient was given bolus injection twice to induce anesthesia, first at $t=3$ minute with $20 \mathrm{mcg}$ and then at $t=5$ minute with $20 \mathrm{mcg}$. The surgical procedures were manually recorded. Three major types of stimulation were identified: (1) During the initial drug administration (the first 6 minutes), due to set-up stimulation and patient nervousness. (2) Incision at $t=45$ minute for about 5 minutes duration. (3) Closing near the end of the surgery at $t=60$ minute.

The data from the first 30 minutes and in the interval where the bolus and stimulation impact was minimal (between $t=10$ to $t=30 \mathrm{~min}$.) were used to determine model parameters and function forms. By optimal data fitting using the least-squares method [18], we derived the estimated parameter values. For this data case, the patient sensitivity function shown in Fig. (9). Under a sampling interval $T=1$ second, which was the standard data transfer interval for the BIS monitor, the combined linear dynamics was estimated. The z-transfer function of the patient model with propofol infusion rate as the input and BIS measurement as the output was identified as

$$
P(z)=\frac{0.01872 z^{2}-0.08813 z+0.09016}{z^{5}-1.159 z^{4}+0.7501 z^{3}-0.5989 z^{2}+0.2984 z-0.2678}
$$

with sampling interval $T=1$ second.

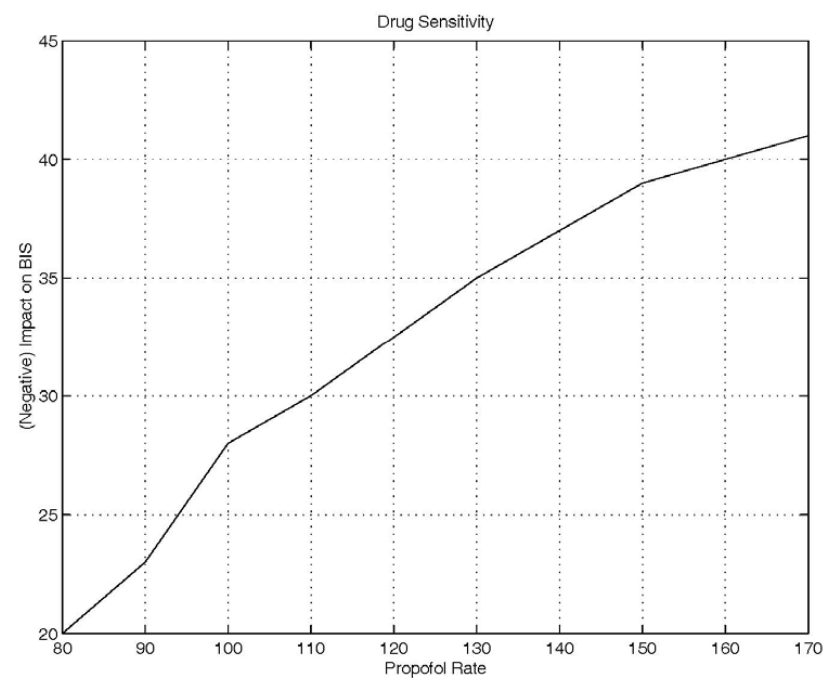

Fig. (9). Drug sensitivity function (Titration).

The actual BIS response was then compared to the model response over the entire surgical procedure. Comparison results are demonstrated in Fig. (10). Here, the inputs of titration and bolus were the recorded real-time data. The model output represented the patient response very well. In particular, the model captured the key trends and magnitudes of the BIS variations in the surgical procedure. This indicated that the model structure contained sufficient freedom in representing the main features of the patient response.

\subsection{Simplified System Functions}

The linear patient dynamics in (1) could be approximated by a simple system. The plant in this case was identified as a 5 th order difference equation in (1). The system could be well approximated by a continuous-time system that consisted of a pure time delay and a first-order dynamics, sampled with sampling interval $T=1$ second. The continuous-time system's transfer function was estimated by using the least-squares algorithm as

$P(s)=e^{-5 s} \frac{0.93}{73 s+1}$.

The step responses of the original system and the simplified system $P(s)$ are shown in Fig. (11). Since this 

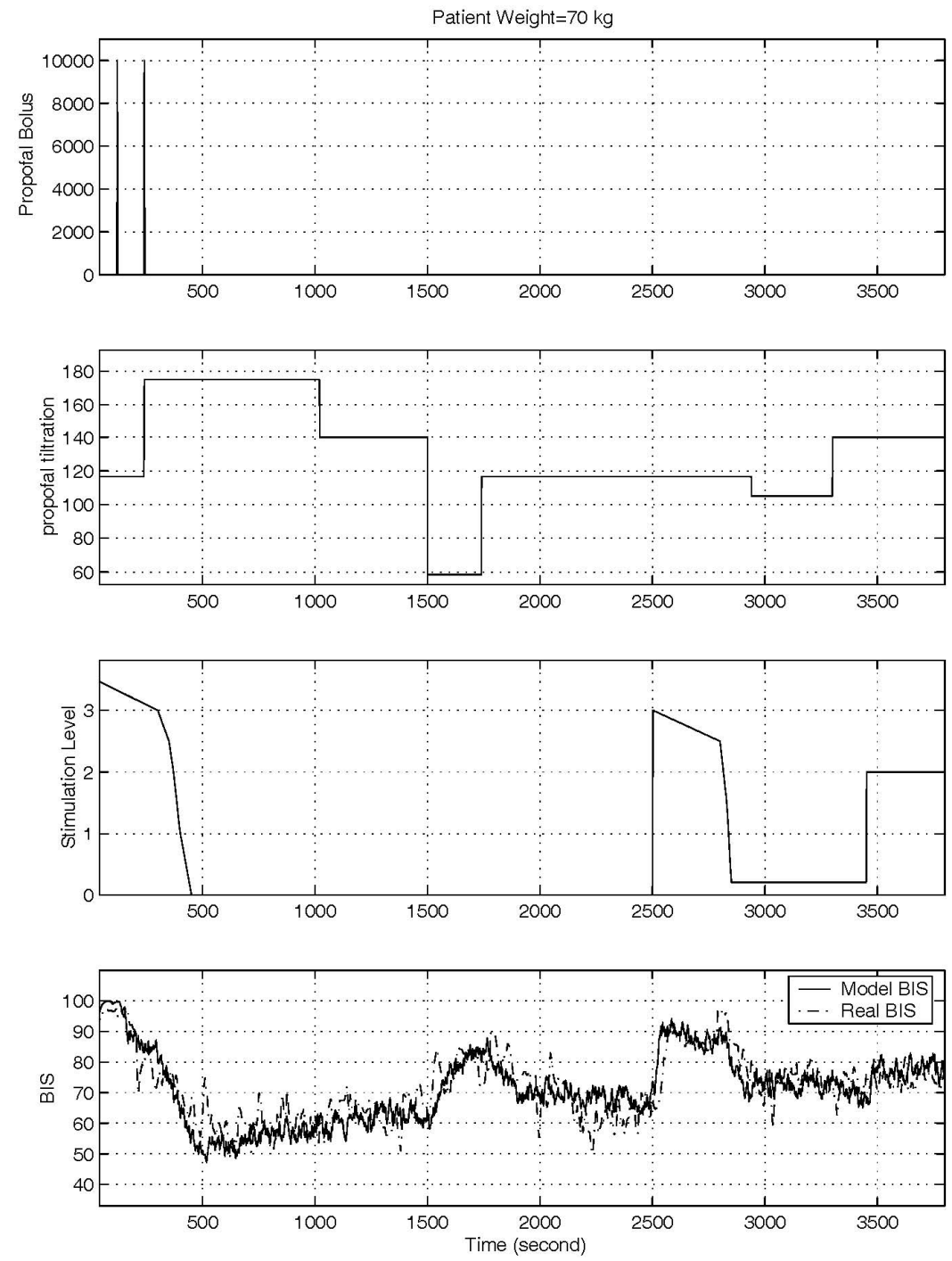

Fig. (10). Actual and model responses.

model contained only three parameters, it was much easier to be identified in real time.

It is also possible to use a simplified nonlinear function which has only three parameter $r, \alpha, b$ to represent the sensitivity function $f$ :

$$
y=r\left[u \pm\left(\frac{\operatorname{erf}(\alpha u)}{\operatorname{erf}(\alpha b)}-u\right)\right]
$$

This function can be linear or nonlinear which is determined by the sign of \pm . Fig. (12) shows an example of this function.

\subsection{MIMO Patient Modeling}

In principle the above SISO method can be employed in MIMO models, by considering an $m$-input and $n$-output system as a collection of $m \times n$ subsystems, each of which represents one input and one outcome relationship. For example, if two drugs (propofol and fentanyl) are present and three outcomes (depth, blood pressures, and heart rates) are considered, we may view this as a collection of 6 subsystems, including propofol-to-depth, propofol-to-BP, propofol-to-HR, fentanyl-to-depth, fentanyl-to-BP, fentanylto-HR subsystems. This approach, however, involves many

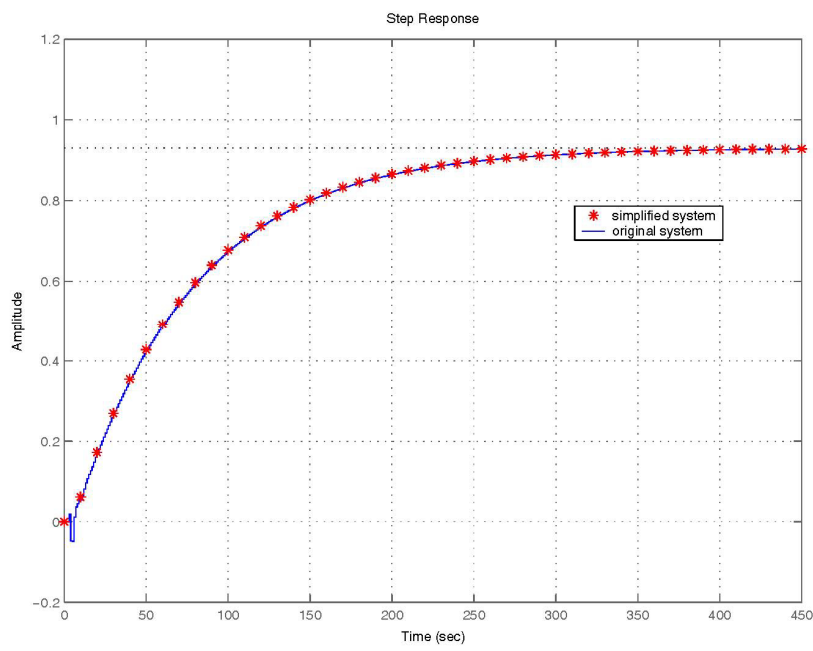

Fig. (11). Step responses of the original system and the simplified system. 
model parameters and encounters high system complexity in modeling processes. For example, if each submodel contains only $L$ parameters, the over system will have $6 L$ parameters that must be updated in real time, which is a substantial complexity in this application.

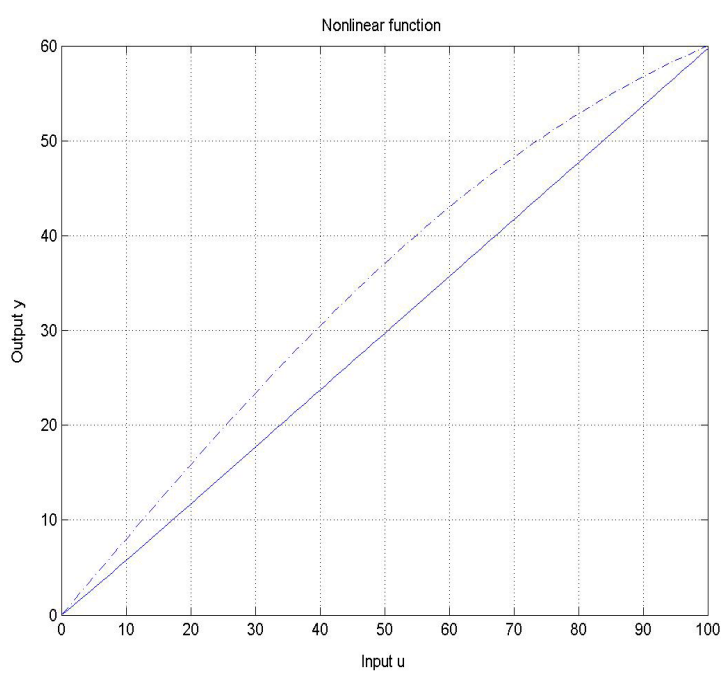

Fig. (12). The sensitivity function is simplified by a nonlinear function which has three parameters: $r, \alpha, b$. The sign of \pm determines the function's shape.

Modifications to the above approach were made to reduce modeling complexity by the following combination method. Since both propofol and fentanyl went through similar propagation and metabolism to influence blood pressure and heart rate, it was reasonable to use the same time delay and same dynamic response speed for both models. They, however, demonstrated very different sensitivity [19]. As a result, it was reasonable to use only one scaling factor to represent the difference between propofol and fentanyl in their impact on the blood pressure and heart rate. Furthermore, fentanyl did not have influence on BIS index [19]. This method reduced significantly the number of model parameters. For example, if each model contained $L$ parameters, in the case of two drugs and three outcomes, this method would reduce the number of parameters from $6 L$ to $3 L+3$. For this application, we had $L=11$ ( 8 parameters for the 5 th order linear system and 3 for the nonlinear part) for the initial model structure; or $L=6$ (6 parameters for the simplified delay system and 3 for the nonlinear part) after simplification. The above method of combining submodels reduced model complexity from $6 L=66$ to $3 L+3=36$ for the initial model structure; or from $6 L=36$ to $3 L+3=21$ for the simplified delay system. These complexity reductions were substantial in making real-time MIMO modeling a feasible option in anesthesia applications which were not data rich.

\section{MULTI-OBJECTIVE ANESTHESIA PREDICTIVE DIAGNOSIS}

Here, we considered a special case that involved two outcomes: the anesthesia depth $y_{B}$ and and mean blood pressure $y_{P}$. The continuous control was provided by propofol titration whose rate was denoted by $u$. Propofol or fentanyl bolus injections could be used when necessary to assist. Also, blood pressures might also be reduced by vasodilation agents or other means if necessary.

From a system viewpoint, we had a system with two types of control inputs: one main control variable $u$ that was continuously managed, and another pulse types of control $v$ that was used only when it was needed. The system had two outputs $y_{B}$ and $y_{P}$. The basic strategy was to use $u$ to achieve control objectives as much as possible. When $u$ alone could not achieve certain control objectives, $v$ was used to assist $u$ to reach the goal.

This paper was focused on predictive diagnosis: (1) Given the current input $u$, what would be the outcomes in the near future? (2) If the input was changed to a new value, what would be the impact of this change? (3) If we wanted the outcomes to settle at a new level, would it be possible to achieve it with assistance from $v$ ?

\subsection{Basic Ideas and Analysis}

We first considered a patient whose BIS response to propofol titration rate $u(\mathrm{mcg} / \mathrm{kg} / \mathrm{min})$ was modeled by the transfer function

$$
x_{B}=e^{-\tau_{B} s} \frac{K_{B}}{T_{B} s+1} U(s) ; \quad y_{B}=100-f_{B}\left(x_{B}(t)\right)+d_{B}
$$

where $\tau_{B}$ was the initial delay, $K_{B}$ was the drug sensitivity, $T_{B}$ represented the response speed of the patient, $f_{B}$ was a nonlinear sensitivity function, and $d_{B}$ was an external disturbance to the BIS value; and $U(s)$ was the Laplace transform of input drug rate; and whose mean blood pressure response to propofol titration was represented by the simplified delay model

$$
x_{P}=e^{-\tau_{P} s} \frac{K_{P}}{T_{P} s+1} U(s) ; \quad y_{P}=110-f_{P}\left(x_{P}(t)\right)+d_{P}
$$

where those parameters had the same meanings as in the BIS model.

We used $w(t)=\left[y_{B}(t), y_{P}(t)\right]$ to represent the outputs. In real implementations of our prediction algorithms, the patient models would be generated in real-time, using actual input-output data. Here, for methodology description we used the above models to show how outcome prediction was performed. Although several methods, such as artifical neural networks, could also perform time series prediction, they carry far more complexity than our model structures. For anesthesia applications, our method had two advantages: (1) The model introduced in this paper was simple in structure and contained less numbers of system parameters without sacrificing much accuracy. As a result, it was easy to be identified in real time. (2) Each parameter in the model had physiological meanings that could be understaood by an anesthesiologist. 
The output vector $w(t)$ was initially at an equilibrium point with $w\left(t_{0}\right)=\left[y_{B}\left(t_{0}\right), y_{P}\left(t_{0}\right)\right]$ and input $u\left(t_{0}\right)=u_{0}$. When $u(t)$ was increased from $u_{0}$ to $u_{0}+\Delta$, the outcome $w(t)$ started to change due to this input jump. Outcome prediction showed how $w(t)$ would change in the near future and where it would settle to a new equilibrium. When the patient model was available, outcome prediction could be calculated from the model as follows.

$$
\text { From } x_{B}=e^{-\tau_{B} s} \frac{K_{B}}{T_{B} s+1} U(s) \text {, the response } \tilde{x}_{B}(t) \text { to } \Delta
$$

jump at $t_{0}$ was calculated to be: $\tilde{x}_{B}(t)=0, t-t_{0} \leq \tau_{B}$, and $\tilde{x}_{B}(t)=K_{B}\left(1-e^{-\left(t-t_{0}-\tau_{B}\right) / T_{B}}\right) \Delta, t-t_{0}>\tau_{B}$. As a result, for $t>t_{0}$, we had

$x_{B}(t)=x_{B}\left(t_{0}\right)+\tilde{x}_{B}(t)$

and

$$
y_{B}(t)=100-f_{B}\left(x_{B}(t)\right)=100-f_{B}\left(x_{B}\left(t_{0}\right)+\tilde{x}_{B}(t)\right)
$$

Furthermore, $y_{B}(t)$ would settle at the new equilibrium value $100-f_{B}\left(x_{B}\left(t_{0}\right)+K_{B} \Delta\right)$. Following the same analysis, we had also

$\tilde{x}_{P}(t)= \begin{cases}0, & t-t_{0} \leq \tau_{P} \\ K_{P}\left(1-e^{-\left(t-t_{0}-\tau_{P}\right) / T_{P}}\right) \Delta, & t-t_{0}>\tau_{P} .\end{cases}$

As a result, for $t>t_{0}$, we obtained

$x_{P}(t)=x_{P}\left(t_{0}\right)+\tilde{x}_{P}(t)$

and

$y_{P}(t)=110-f_{P}\left(x_{P}(t)\right)=110-f_{P}\left(x_{P}\left(t_{0}\right)+\tilde{x}_{P}(t)\right)$

and $y_{P}(t)$ would settle at the new equilibrium value $110-f_{P}\left(x_{P}\left(t_{0}\right)+K_{P} \Delta\right)$.

The models were used in the following capacity to assist an anesthesiologist to make decisions in anesthesia administration.

\section{Drug Impact Prediction}

Drug impact prediction was an extension of outcome prediction. The outcome prediction provided the future outcome trajectories when one drug decision was made and implemented. Drug impact prediction was an assessment of future outcomes when several drug decisions were being considered. This prediction capability allowed an anesthesiologist to evaluate and decide the optimal choices.

\section{Reachable Sets}

Suppose that the output vector $w(t)$ was initially at an equilibrium point $w\left(t_{0}\right)=w_{0}$. The question here was to determine if the propofol titration control alone was sufficient to achieve a designated target $w_{f}$. If the answer was affirmative, then assistance from $v$ was not needed. Otherwise, $v$ must be used such that after applying a bolus injection $v, w_{f}$ became reachable. The reachable set of the outputs under one drug actions exhausted all possible values of that drug and determined the set of the outputs that could be reached. If the desired outputs were outside this reachable set, the second drug, in our case it was either the fentanyl bolus or propofol bolus, must be used so that the new reachable set contained the desired output values.

\subsection{Case Studies}

To demonstrate our ideas presented in the previous sections, clinical data were collected and analyzed, as detailed in Section 2. One of the case data sets, shown in Fig. (3), was used here. The three inputs included propofol titration, propofol bolus injection, fentanyl bolus injection. The two outputs were the BIS index and MAP. Since the fentanyl bolus had very small impact on the BIS index, we neglected the submodel from the fentanyl bolus to the BIS index. As a result, there were a total of 5 submodels: propofol titration to BIS and MAP, propofol bolus to BIS and MAP, and fentanyl bolus to MAP.

The patient data were used to identify these models, with the identified model listed below.

1. BIS to propofol titration: $x_{B}(s)=e^{-3 s} \frac{0.0163}{46 s+1} U(s)$

$$
y_{B}(t)=100-9 *\left(x_{B}(t)-\left(\operatorname{erf}\left(0.4 * x_{B}(t)\right)-x_{B}(t)\right)\right)
$$

2. BIS to propofol bolus: $y_{B}(s)=100-e^{-15 s} \frac{200}{2000 s+1} U(s)$

3. MAP to propofol titration:

$$
y_{P}(s)=110-e^{-250 s} \frac{0.1}{200 s+1} U(s)
$$

4. MAP to propofol bolus:

$$
y_{P}(s)=110-e^{-25 s} \frac{42}{4000 s+1} U(s)
$$

5. MAP to fentanyl bolus:

$$
y_{P}(s)=110-e^{-100 s} \frac{80}{4000 s+1} U(s)
$$

Fig. (13) illustrates the identified model outputs with the real patient outcomes. The models captured the main trends of the BIS and MAP quite well. We should emphasize that this was achieved with a very low model complexity. This trend information was similar to what an anesthesiologist usually required in making anesthesia drug administration decisions.

The models can be used for drug impact prediction. For example, suppose the propofol rate is increased by $30 \mathrm{mcg} / \mathrm{min}$ at $t_{0}=80 \mathrm{~second}$. Fig. (14) shows how this drug infusion rate change affected the BIS value and MAP. In any time instant, to provide decision assistance to an anesthesiologist, different drug infusion strategies could be 
considered and their impact on the outputs was plotted to evaluate and compare consequences of such actions in the near future. For example, to understand drug impacts prediction of increasing propofol rates by 10,20,30,40,50, we plotted all these cases simultaneously. These impact predictions are plotted in Fig. (15).

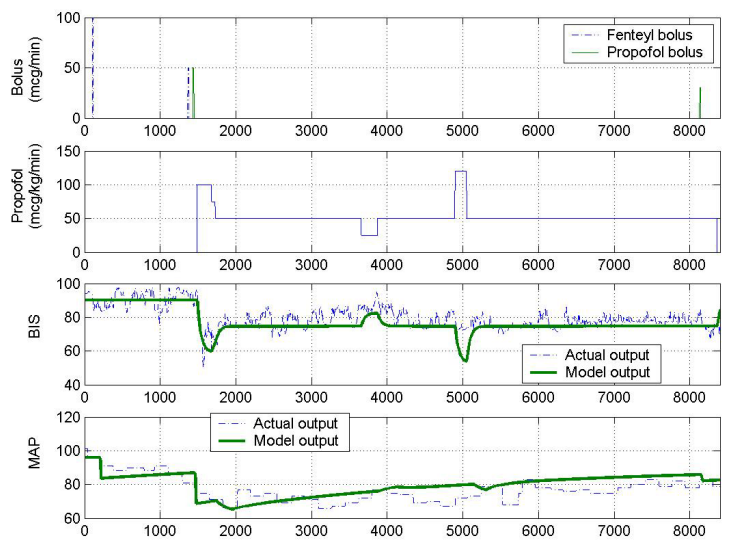

Fig. (13). Multi-input-multi-output patient model.
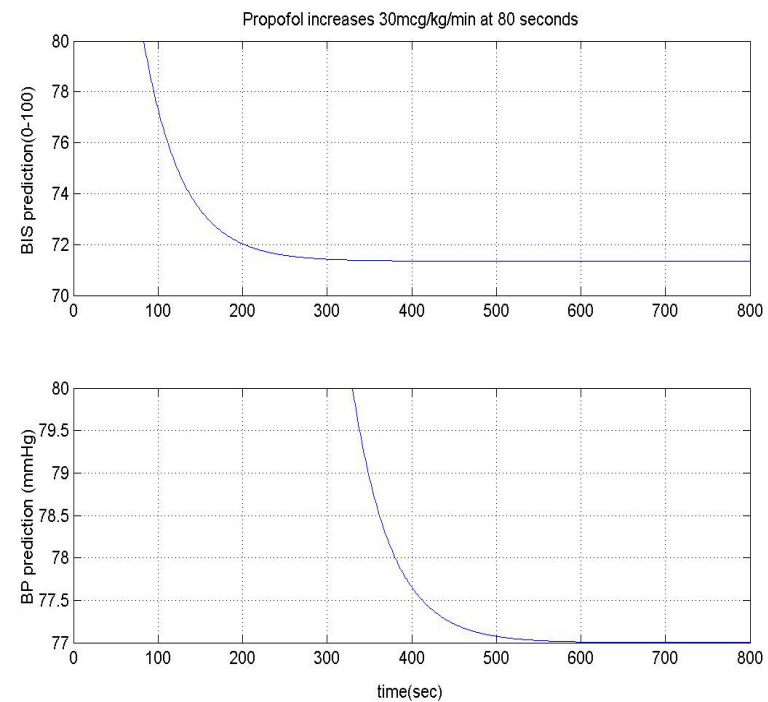

Fig. (14). Outcome predictions.

To study the reachable sets, suppose at a given time the BIS index was 70 and the MAP was $80 \mathrm{mmHg}$. Fig. (16) shows all potential patient steady-state outcome sets when various drugs were administrated. From Fig. (16), different designated targets could be achieved through administrating different drugs. For example, to depress the patient blood pressure without changing BIS values, only fentanyl bolus was needed. But, to push the BIS value to a low level of 60 without much effect on blood pressures (mean arterial pressure of $80 \mathrm{mmHg}$ was usually the desired level during anesthesia), it would be better to use propofol bolus than propofol titration. This was reflected in the reachable set of propofol bolus that had less impact on the MAP. We should also point out that one may also use propofol bolus with a reduced propofol titration to keep MAP unchanged.
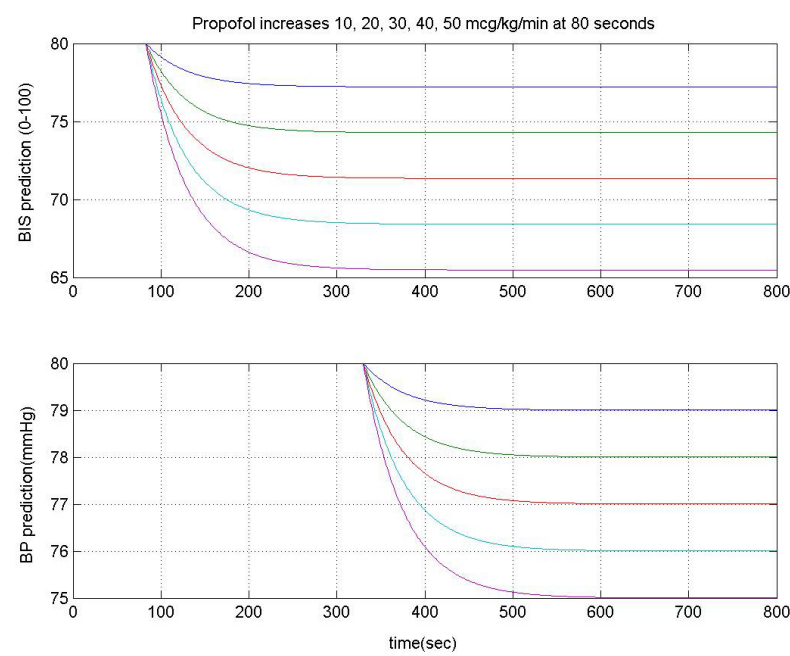

Fig. (15). Drug impact prediction.

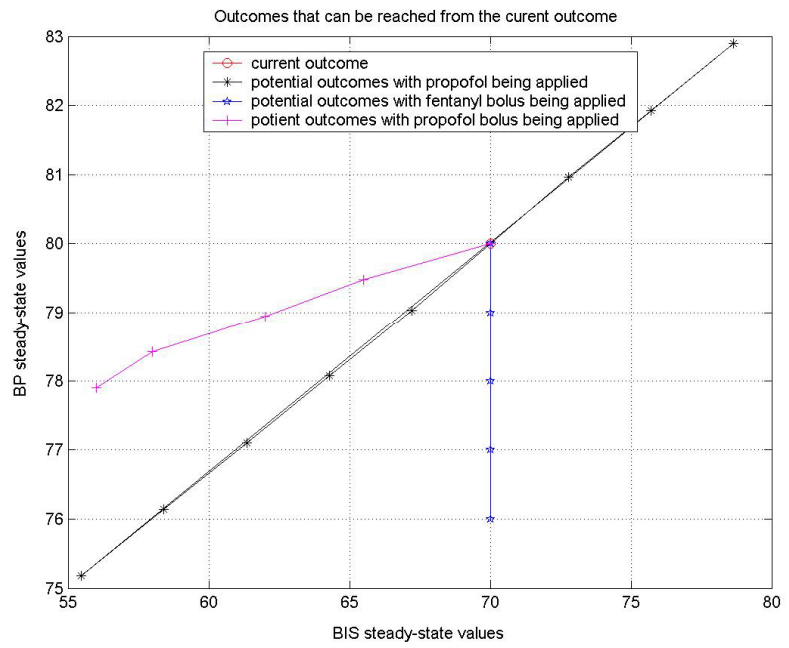

Fig. (16). Reachable outcomes from the current outcome with different drugs inputs.

\section{DISCUSSIONS AND CONCLUSIONS}

This paper investigated the problem of real-time monitoring, diagnosing, and predicting multiple outcomes of anesthesia patients. For enhanced anesthesia management, it is essential to view the anesthesia patient dynamics as a multi-input (multi drugs) and multi-output (multi outcomes) system. For predictive diagnosis and decision assistance, a simplified Wiener model structure was introduced and studied for its suitability in representing the patient responses to drug infusion. Furthermore, a method of consolidating submodels was introduced which could significantly reduce the total number of MIMO system parameters. The identified models were shown to have significant utility in anesthesia decision assistance, by developing outcome impact analysis and reachable sets.

A traditional modeling paradigm for describing the releationship between input drugs and patient responses is the pharmacokinetic-pharmacodynamic (PKPD) compartmental model structure. Pharmacokinetics concerns the 
dynamic process of drug distribution in the body, and pharmacodynamics decribes the effects of the drug on the body. The most frequently used PKPD model structure was a three-compartment model published in [20]. PKPD models have been used for anesthesia controller design and monitoring $[1-5,7]$. An alternative modeling methodology was physiological models which further detailed physiological processes to complement simple compartmental models [21]. Both model structures aimed to derive generic models for a targeted patient population. Due to their high complexities, deriving model parameters required a large data set. As a result, they were mostly useful for off line modeling, or at most partial parameter updating online with some selected parameters $[1,3]$. Consequently, these models were not individually tuned for a patient. Our approach aimed to use real-time data on a specific patient to derive individualized model for that patient. This can potentially provide more accurate models since patien-to-patient variations are substantial.

For multi-objective anesthesia diagnosis, several researchers considered multivariate models [14,15,22]. In [14,22], the neural-fuzzy systems were used to model patient dynamics. In [15] a multi-variable piecewise-linear model was used to relate drugs and surgical stimuliations to patient outcomes such as heart rate, BIS index, and blood pressure. However, neural-fuzzy systems are black-box models whose system parameters do not carry clear physiological meanings. This implies that an anesthesiologist won't be able to provide direct inputs to adjust or limit model parameters. Furthermore, the models in $[14,15,22]$ contained many parameters and hence were highly complex. The initial learning phase for neural-fuzzy systems is usually time consuming which limits their utility in using small real-time data sets to derive a reliable and individualized patient model. Our approach used a simplified model structure that contained only 3-4 parameters that reflected an anesthsiologist's understanding of a patient's dynamic response, such as time delay, speed, and sensitivity. Consequently, his/her knowledge could be used to adjust or bound parameters. The reduced parameter set made it suitable for real-time multiobjective patient modeling due to its reduced complexity.

\section{REFERENCES}

[1] V. Sartori, P.M. Schumacher, T. Bouillon, M. Luginbuehl, and M. Morari, On-line estimation of propofol pharmacodynamic parameter, Conf. Proc. IEEE Engineering in Medicine and Biology Society, September 1-4, 2005, Shanghai, China, 2005.

[2] A. Gentilini, M. Rossoni-Gerosa, C.W. Frei, R. Wymann, M. Morari, A.M. Zbinden, and T.W. Schnider, "Modeling and closedloop control of hypnosis by means of bispectral index (BIS) with isoflurane," IEEE Transactions on Biomedical Engineering, vol. 48, no. 8, pp. 874-889, 2001.

[3] E. Furutani, Y. Sawaguchi, G. Shirakami, M. Araki, K. Fukuda, A hypnosis control system using a model predictive controller with online identification of individual parameters, 2005 IEEE Conference on Contr., August 28-31, 2005, Toronto, Canada, 2005. Proc. of the Anaesthetic Research Society, Nov. 11-12, 1999 ,
Edinburg, 1999.

[5] J.C. Eisenach, "Reports of Scientific Meetings - Workshop on Safe Feedback Control of Anesthetic Drug Delivery," Anesthesilogy, vol. 91, pp: 600-601, 1999.

[6] D.A. Linkens, "Adaptive and Intelligent Control in Anesthesia," IEEE Control Systems Magazine, vol. 12, pp. 6-11, Dec. 1992.

[7] X.-S. Zhang, R.J. Roy, and J.W. Huang, Closed-loop System for Total Intravenous Anesthesia by Simultaneously Administering Two Anesthetic Drugs, Proc. of the 20th Annual International Conference of the IEEE Engineering in Medicine and Biology, Oct. 29-Nov. 1, 1998, Hong Kong, China, 1998.

[8] L.Y. Wang, and H. Wang, "Control-oriented modeling of BISbased patient response to anesthesia infusion," In: 2002 Internat. Conf. Math. Eng. Techniques in Medicine and Bio. Sci., June 2427, 2002, Las Vegas, 2002.

[9] L.Y. Wang, and H. Wang, "Feedback and predictive control of anesthesia infusion using control-oriented patient models,'In: 2002 Internat. Conf. Math. Eng. Techniques in Medicine and Bio. Sci., June 24-27, 2002, Las Vegas, 2002.

[10] L.Y. Wang, H. Wang, and G. Yin, "Anesthesia infusion models: Knowledge-based real-time identification via stochastic approximation," In: 41st IEEE Cont. and Dec. Conf., Dec. 10-13, 2002, Las Vegas, 2002.

[11] L.Y. Wang, H. Wang, and G. Yin, "Reliable nonlinear identification in medical applications," In: 13th IFAC Symposium on System Identification (SYSID 03), Aug. 27-29, 2003, Rotterdam, The Netherlands, 2003.

[12] T.J. Gan, P.S. Glass, A. Windsor, F. Payne, C. Rosow, P. Sebel, and P. Manberg, "Bispectral index monitoring allows faster emergence and improved recovery from propofol, alfentanil, and nitrous oxide anesthesia," Anesthesiology, vol. 87, pp. 808-815, 1997.

[13] C. Rosow, and P.J. Manberg, "Bispectral Index Monitoring," Annual of Anesthetic Pharmacology, vol. 2, pp. 1084-2098, 1998.

[14] M. Mahfouf, C. Nunes, D. Linkens, and J. Peacock, "Modeling and multivariable control in anesthesia using neural-fuzzy paradigms Part II. Close-loop control of simultaneous administration of propofol and remifentanil," Artificial Intelligence in Medicine, vol. 35, no. 3, pp. 207-213, 2005

[15] H. H. Lin, C.L. Beck, and M. J. Bloom, "On the use of multivariable piecewise-linear models for predicting human response to anes-thesia," IEEE transactions on Bio. Eng., vol. 51, no. 11, pp. 1876-1887, Nov. 2004.

[16] J. S. Shieh, M. F. Abbod, C. Y. Hsu, S. J. Huang, Y. Y. Han and S. Z. Fan, "Monitoring and Control of Anesthesia Using Multivariable Self-Organizing Fuzzy Logic Structure," Fuzzy Systems in Bioinformatics and Computational Biology, vol. 242, pp. 273-295, 2009.

[17] Y. Sreenivas., S. Lakshminarayanan, and G. P. Rangaiah, Automatic regulation of anesthesia by simultaneous administration of two anesthetic drugs using model predictive control, World Congress on Medical Physics and Biomedical Engineering, vol. 14, pp. 82-86, Coex Seoul, Korea, 2007.

[18] L. Ljung, and T. Söderström, Theory and Practice of Recursive Identification. MIT Press: Cambridge, MA, 1983.

[19] M. Nakayama, H. Ichinose, S. Yamamoto, N. Kanaya, and A. Namiki, "The effect of Fentanyl on hemodynamics and bispectral index changes during anesthesia induction with propofol," Journal of Clinical Anesthesia, vol. 14, pp. 146-149, 2002.

[20] J. Schüttler, and H. Ihmsen, "Population pharmacokinetics of propofol: a multicenter study," Anesthesiology, vol. 92, pp. 727$738,2000$.

[21] K. Stadler, "Modeling and control in anesthesia from design to validation", $\mathrm{PhD}$ Thesis, ETH Zyrich, Institutfur Automatik Publikation, 2003.

[22] C. Nunes, M. Mahfouf, D. Linkens, and J. Peacock, "Modeling and multivariable control in anesthesia using neural-fuzzy paradigms Part I. Classification of depth of anesthesia and development of a patient model," Artificial Intelligence in Medicine, vol. 35, no. 3, pp. 195-206, 2005.

Received: February 17, 2010

(C) Tan et al.; Licensee Bentham Open.

This is an open access article licensed under the terms of the Creative Commons Attribution Non-Commercial License (http://creativecommons.org/licenses/by-nc/3.0/) which permits unrestricted, non-commercial use, distribution and reproduction in any medium, provided the work is properly cited. 